\title{
Consolidation Clinical Trial Setting
}

National Cancer Institute

\section{Source}

National Cancer Institute. Consolidation Clinical Trial Setting. NCI Thesaurus. Code C156599.

A clinical trial or treatment setting in which the therapy of interest is considered consolidation therapy. 